\title{
A systematic review and meta-analysis of Liuzijue in stable patients with chronic obstructive pulmonary disease
}

\author{
Lu Xiao ${ }^{1,2}$, Hongxia Duan ${ }^{1}$, Peijun $\mathrm{Li}^{3}$, Weibing Wu${ }^{3}$, Chunlei Shan ${ }^{1,4}$ and Xiaodan Liu ${ }^{1,4^{*}}$
}

\begin{abstract}
Background: To investigate the effectiveness of Liuzijue exercise on chronic obstructive pulmonary disease (COPD) in the stable phase.

Methods: We searched six electronic bibliographic databases (PubMed, EMBASE, The Cochrane Library, Web of Science, CNKI, and Wan Fang Data) from inception to August 2018. Randomized controlled trials (RCTs) were included if they evaluated the effect of Liuzijue exercise on stable COPD. Cochrane Collaboration risk-of-bias tool (Cochrane Handbook 5.1.0) was used to assess the risk of bias of included RCTs. Meta-analysis was performed using the Review Manager software (RevMan V.5.3.5) provided by the Cochrane Collaboration. Outcomes assessed included dyspnea, exercise capacity, lung function, and quality of life.
\end{abstract}

Results: Fourteen RCTs involving 920 stable COPD patients were included in this systematic review and meta-analysis. The control groups received usual care. The average number of training sessions per participant was 9.3 per week, and the average length of these training sessions was 31.6 min per week. Training duration varied from 3 to 12 months. Metaanalysis results showed that Liuzijue exercise can effectively improve patients' Modified Medical Research Council Dyspnea Scale scores ( $\mathrm{MD}=-0.73,95 \% \mathrm{Cl}:-1.13$ to $-0.33, P<0.05), 6 \mathrm{MWD}(\mathrm{MD}=17.78,95 \% \mathrm{Cl}: 7.97$ to $27.58, P<0.05)$, forced expiratory volume in one second $\left(F E V_{1}\right)(\mathrm{MD}=0.23,95 \% \mathrm{Cl}: 0.07$ to $0.38, P<0.05)$, the percentage of predicted values of $\mathrm{FEV}_{1}\left(\mathrm{FEV}_{1} \%\right.$ pred) (MD $=7.59,95 \% \mathrm{Cl}: 2.92$ to 12.26, $\left.P<0.05\right)$, FEV 1 /FVC (Forced vital capacity) ratio (MD =6.81, 95\% Cl: 3.22 to $10.40, P<0.05)$, Quality of life: St. George's Respiratory Questionnaire total score (MD $=-9.85,95 \% \mathrm{Cl}:-13.13$ to -6.56 , $P<0.05)$, and Chronic Obstructive Pulmonary Disease Assessment Test score ( $M D=-2.29,95 \% \mathrm{Cl}:-3.27,-1.30, P<0.05)$.

Conclusion: Evidence from meta-analysis suggested that Liuzijue exercise could improve dyspnea, exercise endurance, lung function, and quality of life for stable COPD patients. However, owing to the methodological bias and the placebo effect of Liuzijue exercise, there is a need for further research to confirm these findings.

Trial registration: PROSPERO (ID: CRD42019130973).

Keywords: Chronic obstructive pulmonary disease, Liuzijue, Exercise

\footnotetext{
* Correspondence: hzhp403@126.com

'School of Rehabilitation Science, Shanghai University of Traditional Chinese Medicine, Shanghai, China

${ }^{4}$ Institute of Rehabilitation Medicine, Shanghai Academy of Traditional Chinese Medicine, Shanghai, China

Full list of author information is available at the end of the article
} 


\section{Background}

Chronic obstructive pulmonary disease (COPD) is a common chronic pulmonary disease with high morbidity and mortality. The characteristics of COPD patients are persistent airflow restriction and respiratory symptoms resulting from airway and/or alveoli abnormalities caused by longterm exposure to noxious particles or gases [1]. A Lancet study showed that the prevalence of COPD has increased from 8.2 to $13.7 \%$ among Chinese people over the age of 40 [2, 3]. In 2016, there were 251 million cases of COPD and about 3 million deaths caused by COPD worldwide [4]; the direct medical cost ranged from 72 to 3563 USD per capita per year [5]. COPD is the fourth leading cause of death in the world at present, and the World Health Organization has predicted that it will become the third leading cause by 2030 [6]. Owing to the high morbidity, mortality, and massive economic burdens it presents society, increasing numbers of researchers are paying close attention to the prevention and treatment of COPD.

Lung function tests are essential for COPD diagnosis. Studies have shown that worsening COPD severity is associated with higher mortality and hospitalization [7]. A low $\mathrm{FEV}_{1}$ (forced expiratory volume in one second)/FVC (Forced vital capacity) ratio is also related to higher mortality [8]. In addition to decrease lung function and longlasting respiratory symptoms, COPD patients may have skeletal muscle dysfunction, such as decreased muscle strength $[9,10]$ and exercise endurance [1]. These symptoms can lead to lower exercise capacity and a worse quality of life. An exercise capacity assessment includes assessing muscle strength and exercise endurance. The sixminute walking test is an efficient tool for assessing exercise endurance, which is closely related to many functional outcomes for COPD patients [11, 12]. St. George's Respiratory Questionnaire (SGRQ) and the Chronic Obstructive Pulmonary Disease Assessment Test (CAT) are common measurements of quality of life. The SGRQ and CAT scores are significantly correlated and both with high reliability and validity [13-15]. An improvement in both scores is significantly correlated with improvements in Modified Medical Research Council Dyspnea Scale (mMRC) score, lung function, and risk of COPD exacerbation [16-21]. However, the correlation between CAT score and pulmonary function, dyspnea, exercise performance, and psychological factors is lower than its correlation with total SGRQ score [14, 19]. The relevance between total SGRQ score and COPD disease severity is higher [22].

The Global Initiative for Chronic Obstructive Lung Disease has identified pulmonary rehabilitation as an essential prevention and control measure for COPD. As the cornerstone of pulmonary rehabilitation, exercise training showed significant improvement for dyspnea, quality of life, exercise endurance, and rate of hospital re-admissions in COPD patients [1]. Traditional Chinese exercises are a type of exercise training that is widely used in pulmonary rehabilitation programs for clinically stable COPD patients. Many clinical studies suggest that traditional Chinese exercise can improve the health of COPD patients [23-25]. Liuzijue is a moderate-low intensity traditional Chinese exercise. Breathing is an important component of Liuzijue; when coordinated with simple body movements, breathing can strengthen the body and improve the bodily function of Liuzijue practitioners. Liuzijue exercises include six pronunciations: 'XU', 'HE', 'HU', 'SI', 'CHUI', and 'XI'. Different pronunciations will produce different sound frequencies and resonate with different parts of the body, which is beneficial for health [26]. It is necessary to adopt long and steady abdominal breathing and pursed-lip breathing when practicing Liuzijue. The movements of Liuzijue contain stretching, flexion, and extension of upper limbs, rotation of the body, expansion of the chest, and contraction and relaxation of the abdomen [27, 28]. Upper limb exercise can improve the lung function, dyspnea, and quality of life of COPD patients $[29,30]$. The vocal organs exert different forces during different pronunciations, and when coordinated with different body movements, can exercise the major and accessory respiratory muscles [31], improve dyspnea, regulate lung function, and increase exercise capacity, eventually improving the patient's quality of life [31, 32]. Based on the characteristics of breathing methods and body movements of Liuzijue, we considered that it may be suitable for the rehabilitation needs of COPD patients.

At present, many clinical studies suggest that traditional Chinese exercise can improve the condition of COPD patients. Many meta-analyses have already [33-36] investigated the effects of multiple traditional Chinese exercises for COPD patients, but meta-analysis about the effects of Liuzijue in COPD patients is still lacking. Therefore, we exclusively searched for and collated relevant Chinese and English studies focusing on Liuzijue exercise. Subsequently, we conducted a systematic review and metaanalysis to clarify whether practicing Liuzijue exercise is beneficial to respiratory symptoms, lung function, exercise capacity, and quality of life in patients with stable COPD.

\section{Methods}

Inclusion and exclusion criteria, as well as analytical methods, were registered in the PROSPERO (http://www. crd.york.ac.uk/PROSPERO) database (PROSPERO number, CRD42019130973).

\section{Selection criteria}

Eligibility criteria: (1) Study type: RCTs published in Chinese and English. (2) Subjects: patients diagnosed with stable COPD. (3) Interventions: the control group was given routine drug treatment and health guidance; the intervention group received Liuzijue or simplified Liuzijue exercise. (4) Outcomes: A. Dyspnea: Medical Research Council Dyspnea 
Scale (MRC) or mMRC; B. Exercise capacity: maximal inspiratory pressure (MIP), maximal expiratory pressure (MEP), limb muscle strength, six-minute walking distance (6MWD), 30 Second Sit-to-Stand Test (30s SST); C. Lung Function: $\mathrm{FEV}_{1}$, the percentage of predicted values of $\mathrm{FEV}_{1}$ (FEV ${ }_{1} \%$ pred), and $\mathrm{FEV}_{1} / \mathrm{FVC}$ ratio $\left(\mathrm{FEV}_{1} / \mathrm{FVC} \%\right)$; D. Quality of life: SGRQ, CAT.

Exclusion criteria: (1) Inconsistent with our intervention measures; (2) outcomes outside the specified range; (3) duplicated data; (4) full-text was not available; (5) not RCT or with inappropriate random method.

\section{Data sources and search strategy}

We searched six databases (PubMed, EMBASE, The Cochrane Library, Web of Science, CNKI, and Wan Fang Data) from inception to August 2018. The search was conducted with the following keywords in Chinese and English: Liuzijue (Qigong OR Qi gong OR chi gong OR chi kung OR traditional Chinese exercises) and Chronic Obstructive Pulmonary Disease (Chronic Airflow Obstruction OR COPD OR Chronic Obstructive Airway Disease OR Chronic Obstructive Lung Disease).

\section{Study selection and data extraction}

Two researchers (LX and HXD) independently extracted data from the selected studies based on the predetermined inclusion and exclusion criteria. Each disagreement was solved with the help of a third reviewer (XDL) when necessary. The following information was extracted from each included study: first author, the year of publication, sample size, average age, proportion of male and female patients, disease severity level, intervention measures, intervention plan for control group and Liuzijue group, and related outcomes (mMRC/MRC score, MIP, MEP, limb muscle strength, $6 \mathrm{MWD}, \mathrm{FEV}_{1}$, $\mathrm{FEV}_{1} \%$ pred, $\mathrm{FEV}_{1} / \mathrm{FVC} \%$, SGRQ, and CAT score).

\section{Assessment of risk of bias}

We used the Cochrane Collaboration risk-of-bias tool (Cochrane Handbook 5.1.0) to assess risk of bias of included RCTs. Potential sources of bias include random sequence generation, allocation concealment, blinding of participants and staff, blinding of outcome assessors, incomplete outcome data, selective reporting, and other biases. Each trial received a study level score of low, high, or unclear risk of bias for each domain. Two authors (LX and HXD) independently conducted this assessment, and discrepancies were resolved through discussion with a third person (PJL).

\section{Data synthesis}

Meta-analysis was performed using the Review Manager statistical software (RevMan V.5.3.5) provided by the Cochrane collaboration. We analyzed the statistics by the mean difference (MD) and 95\% confidence interval (95\% CI). A $P$ value of $<0.05$ was regarded as statistically significant. The heterogeneity of included studies was assessed using $\mathrm{I}^{2}$ statistics. When the $\mathrm{I}^{2}$ statistic value was less than $50 \%$, the random effects model was used; otherwise, the fixed effects model was used to measure outcomes with heterogeneity.

\section{Results}

\section{Search results}

A total of 258 records (145 in Chinese and 113 in English) were initially identified from the six databases. After excluding 100 duplications, 158 studies were chosen for further evaluation. Through screening the titles and abstracts, 110 studies were excluded. We searched for the full text of the remaining 48 studies, and 14 studies were ultimately included while 34 were excluded (inconsistent with intervention measures $=11$, outcomes outside the specified range $=5$, duplicate data $=13$, full text was not available $=3$, not $\mathrm{RCT}$ or with inappropriate random method $=2$ ). Finally, 14 studies were included for our meta-analysis (Fig. 1).

\section{Characteristics of included studies}

Fourteen RCTs [32, 37-49] published from 2009 to 2018 (of which 12 RCTs were published in Chinese [37-48] and two were published in English [32, 49]) were included. A total of 920 patients (control group $=463$, Liuzijue group $=457$ ) were pooled from all the included trials into our final systematic review and meta-analysis. These patients ranged from 39 to 82.26 years old with a COPD severity stage ranging from mild to very severe. The control group received routine drug therapy and health guidance. The average number of training sessions per participant was 9.3 per week and the average length of these training sessions was 31.6 min per week. Training duration ranged from 3 to 12 months. The characteristics of the included studies are shown in Table S1.

\section{Risk of bias}

Details of the risk of bias assessment for the 14 included RCTs are shown in Fig. 2. We used the Cochrane tool (Cochrane Handbook 5.1.0) to assess the risk of bias. The method of random sequence generation, which was either by random number table or computer random number generator, was mentioned in nine studies [32, $38,41,42,44-46,48,49$ ], while other studies did not mention the random method. Two studies $[32,49]$ reported information about allocation concealment. As the Liuzijue exercises require active engagement, blinding of participants and staff was impossible. Only one study reported the blinding of the assessor [49]. Nine studies [32, 38, 39, 44-49] clearly described the reasons and the 


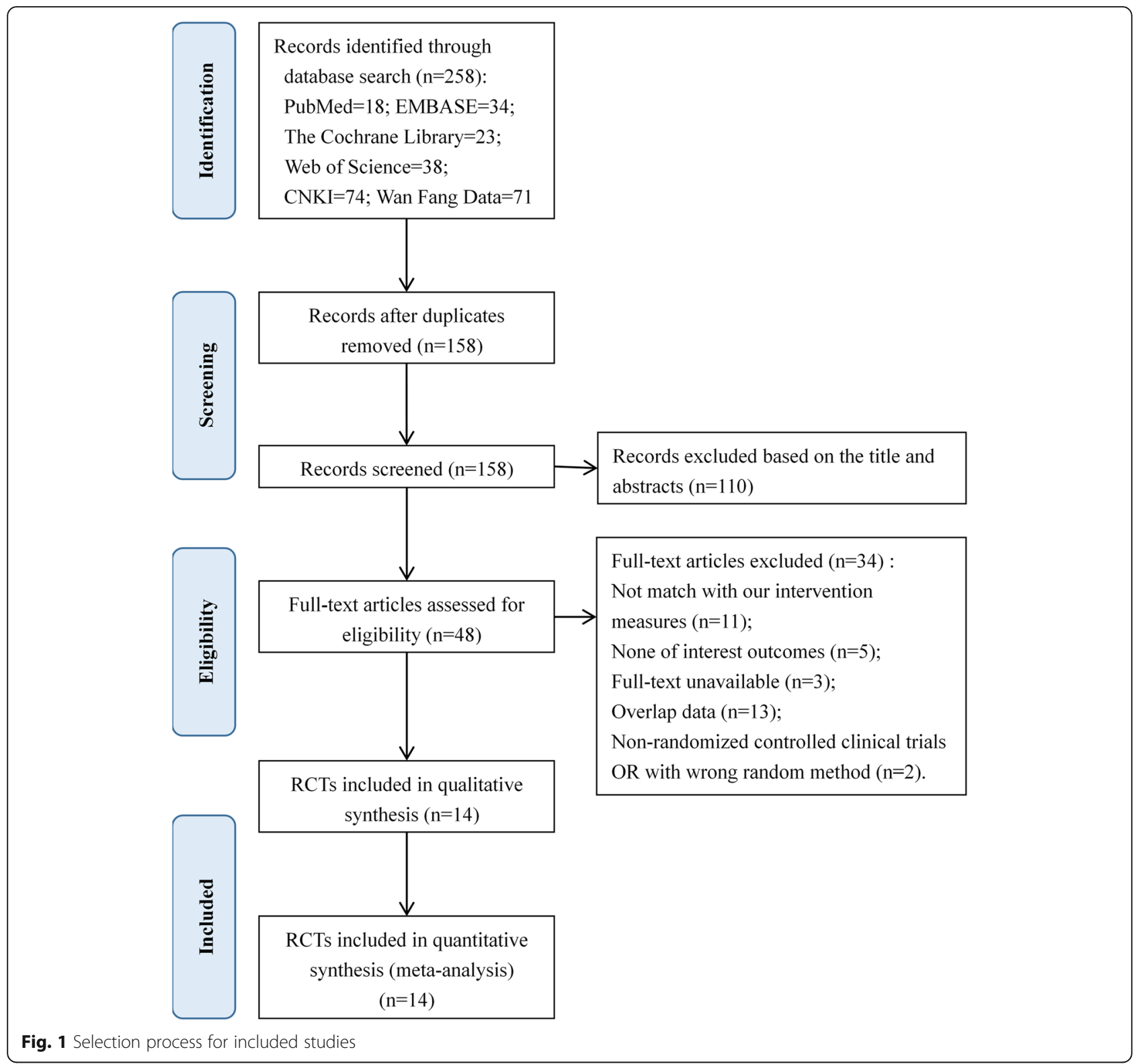

number of exclusion cases. All studies [32, 37-49] have reported all the predefined outcomes.

\section{Meta-analysis results}

\section{Effect of Liuzijue on dyspnea}

MRC (or mMRC) scores were investigated in three included studies [37, 42, 49] (136 COPD patients). Compared with the control group, improvement in MRC (or mMRC) scores in the Liuzijue group was more significant $(\mathrm{MD}=-$ $0.73,95 \%$ CI: -1.13 to $-0.33, \mathrm{I}^{2}=62 \%, P<0.05$, Fig. 3 ).

\section{Effect of Liuzijue on respiratory muscle strength}

MIP and MEP were reported in one study [32]. In the study [32], compared with the control group, the respiratory muscle strength (MIP, MEP) of patients in the Liuzijue group showed significant improvement $(P<0.01)$.

\section{Effect of Liuzijue on limb muscle strength}

Of the 14 studies, only two studies [32, 49] mentioned the assessment of limb muscle strength. A study by Wu et al. [49] reported the efficiency of Liuzijue exercise on patients' handgrip strength. Compared with 6 months prior, the handgrip strength of COPD patients in the Liuzijue group rose an average of $4 \%(P=0.03)$. The results were statistically significant, but there was no significance compared with the control group. Liu et al. [32] reported the effect of Liuzijue on the muscles around the elbow and knee joints. Total work (TW) of the elbow extensor, elbow flexor, and knee extensor, peak torque (PT), and the ratio 

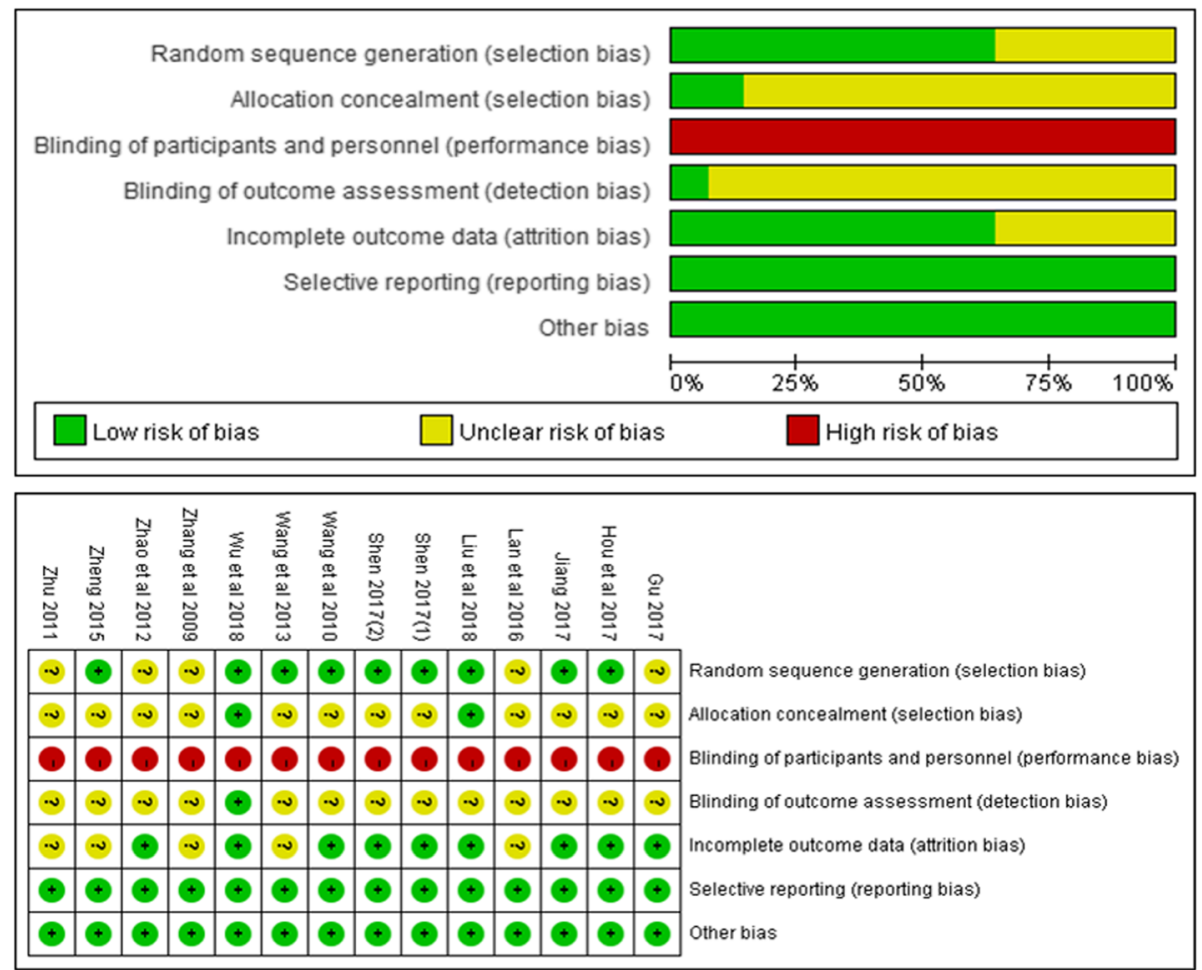

Fig. 2 Assessment of Risk of bias

of peak torque to body weight (PT/BW) of the knee extensor were significantly improved in Liuzijue group after 3 months. The muscle endurance ratio (ER) of the elbow extensor and the TW of the elbow flexor and knee flexor in the Liuzijue group increased significantly compared with the control group $(P<0.05)$.

\section{Effect of Liuzijue on exercise endurance}

Six studies [32, 37, 39, 42, 44, 49] with a total of 274 patients were included in the meta-analysis of 6MWD. The forest plot showed that the 6MWD improvement in the Liuzijue group was significantly greater than that in the control group $\left(\mathrm{MD}=17.78,95 \% \mathrm{CI}: 7.97\right.$ to $27.58, \mathrm{I}^{2}=$ $0 \%, P<0.05$, Fig. 4). In the study by Wu et al. [49], 30s SST was also conducted to evaluate patients' exercise endurance. In this study, $30 \mathrm{~s}$ SST times of the Liuzijue group increased significantly, but there was no significant difference between the two groups.

\section{Effect of Liuzijue on lung function}

Eight studies [37, 39, 40, 44, 45, 47-49] with a total of 502 patients were eligible for $\mathrm{FEV}_{1}$ analysis. Compared with the control group, Liuzijue exercise had a significant effect on $\mathrm{FEV}_{1}$ improvement in COPD patients ( $\mathrm{MD}=0.23,95 \%$ CI: 0.07 to $0.38, \mathrm{I}^{2}=83 \%, P<0.05$, Fig. 5a).

Ten studies [32, 37, 39-44, 46, 49] with a total of 580 patients were included for the $\mathrm{FEV}_{1}$ \%pred analysis. The results showed that the $\mathrm{FEV}_{1}$ \%pred of COPD patients in the Liuzijue group was significantly higher than that in the control group (MD $=7.59,95 \% \mathrm{CI}$ : 2.92 to 12.26 , $\mathrm{I}^{2}=97 \%, P<0.05$, Fig. 5b).

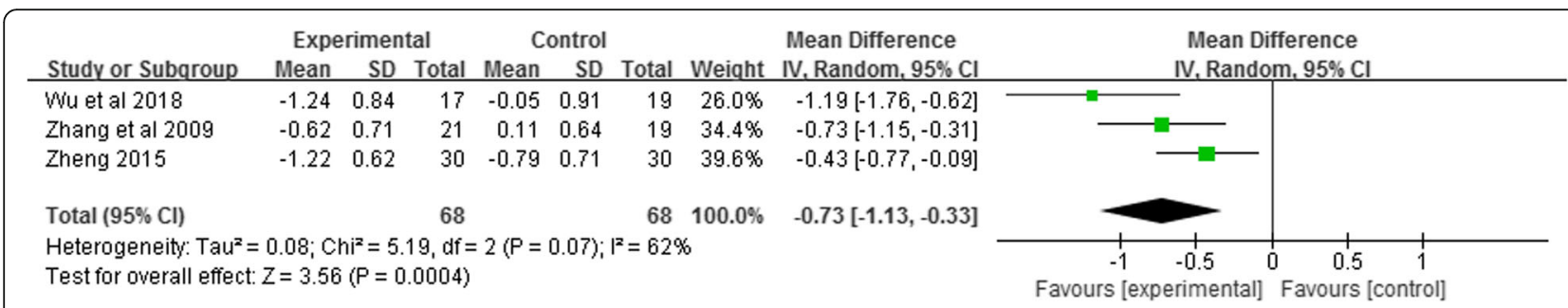

Fig. 3 Meta-analysis of the effect of Liuzijue on MRC (or mMRC). Abbreviations: MRC, Medical Research Council Dyspnea Scale; mMRC, Modified Medical Research Council Dyspnea Scale 


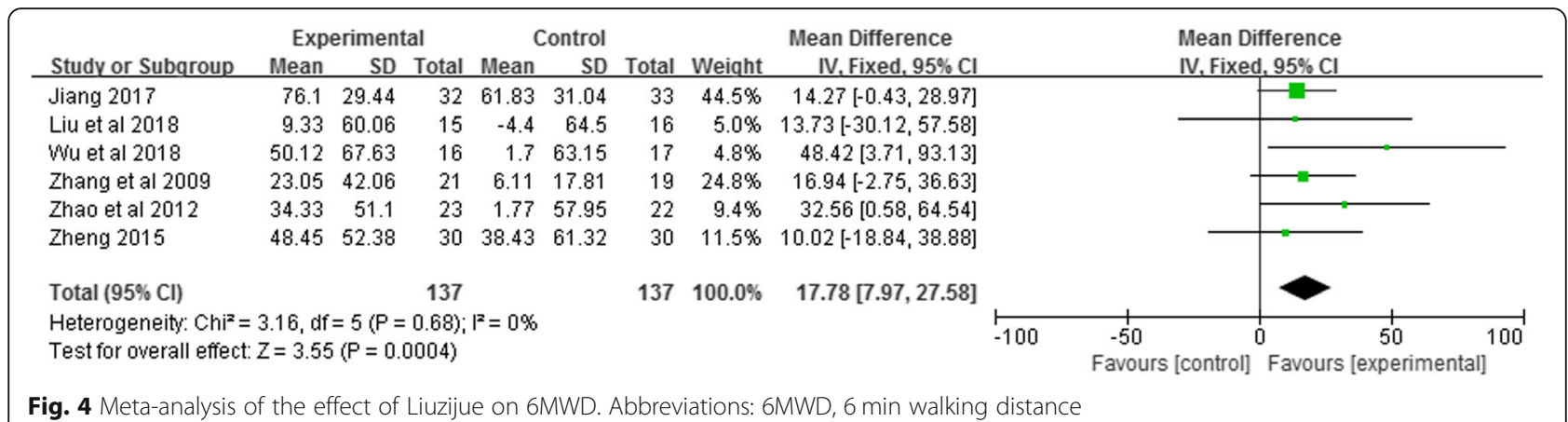

Fig. 4 Meta-analysis of the effect of Liuzijue on 6MWD. Abbreviations: 6MWD, 6 min walking distance

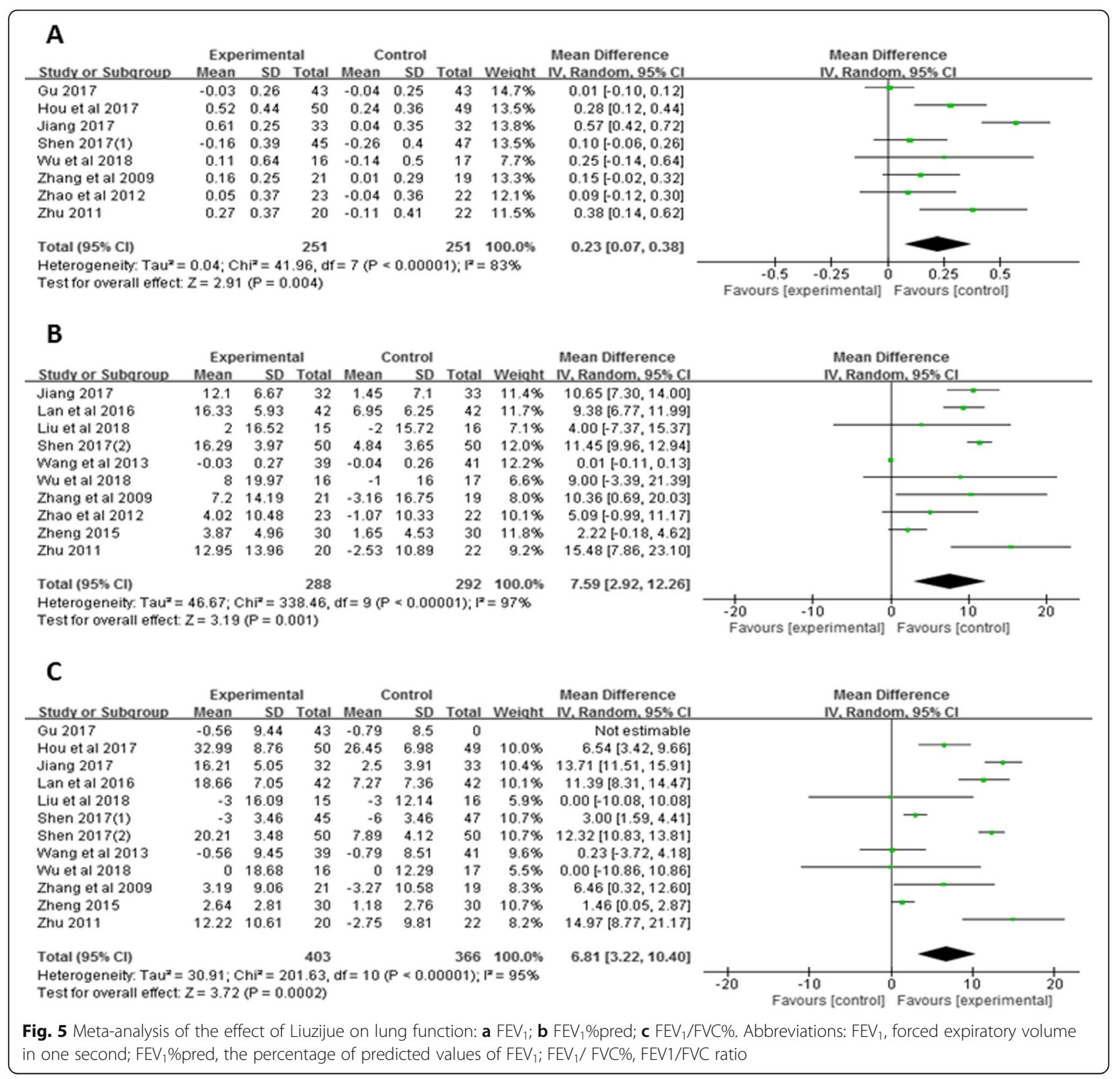


Twelve studies [32, 37, 40-49] with a total of 769 patients were eligible for the analysis of $\mathrm{FEV}_{1} / \mathrm{FVC}_{\%}$. The improvement of $\mathrm{FEV}_{1} / \mathrm{FVC} \%$ in the Liuzijue group was significantly higher than that in the control group $(\mathrm{MD}=$ 6.81, $95 \%$ CI: 3.22 to $10.40, \mathrm{I}^{2}=95 \%, P<0.05$, Fig. 5 c).

\section{Effect of Liuzijue on quality of life}

Among the 14 studies, a total of nine studies assessed quality of life. Five of the nine studies [38, 40, 42, 48, 49] used SGRQ, while four studies [43-46] used CAT.

Five studies [38, 40, 42, 48, 49] (297 COPD patients) that reported SGRQ scores were included in the metaanalysis. This suggests that the total SGRQ scores of patients in the Liuzijue group decreased more significantly $\left(\mathrm{MD}=-9.85,95 \% \mathrm{CI}:-13.13\right.$ to $-6.56, \mathrm{I}^{2}=63 \%, P<$ 0.05, Fig. 6) than those of the control group. Additionally, four [38, 40, 42, 49] (198 COPD patients) of the five studies reported three domains (symptom, activity, and influence) of SGRQ. The results showed that the differences in SGRQ score for symptoms and activity between the two groups were significant $(P<0.05$, Fig. S1).

Four studies [43-46] with a total of 341 COPD patients were included in the meta-analysis of CAT scores. The results showed that the reduction of CAT scores in the Liuzijue group was more significant than that in the control group $\left(\mathrm{MD}=-2.29,95 \% \mathrm{CI}:-3.27,-1.30, \mathrm{I}^{2}=\right.$ $56 \%, P<0.05$, Fig. 7).

\section{Adverse events}

A total of 10 participants across six studies reported adverse events [39, 41, 45, 47-49], while the other studies did not report (Table S2). In the control group, one participant experienced severe pneumonia and respiratory failure [39] and two developed symptoms of herpes zoster $[41,47]$. In the Liuzijue group, two participants died [41, 47], one reported exacerbation of COPD [49], and one was hospitalized due to other medical diseases [45]. There were three participants who experienced other serious diseases [48], but the grouping was unknown. However, there is no indication that these adverse events were caused by the research methods of the six studies.

\section{Discussion}

\section{Dyspnea}

The minimal clinical important difference (MCID) of MRC in patients with COPD is one unit [52]. There were significant clinical differences between two groups in two $[42,49]$ of the three studies. In these two studies, patients practiced Liuzijue at least 5 days $(200 \mathrm{~min})$ per week, and the duration was 6 months. According to the results, we can see that Liuzijue exercise was superior to routine therapy in improving patients' dyspnea. The possible reasons may be as follows: the different forces of the lips, teeth, tongue, and larynx when pronouncing the six different sounds may have exercised patients' respiratory muscles. The main breathing methods of Liuzijue are abdominal breathing and pursed-lip breathing. Pursed-lip breathing can improve dynamic lung hyperinflation, slow down breathing rate, and reduce airway resistance and airway stricture when exhaling, so as to improve patients' dyspnea [53-55]. Moreover, pronunciation when practicing Liuzijue can lengthen the breathing time of COPD patients, which may improve gas retention.

\section{Exercise capacity}

The results of a study by Liu et al. [32] revealed that Liuzijue exercise could significantly improve a patient's respiratory muscle strength (MIP, MEP). However, due to the lack of relevant studies, the improvement of respiratory muscle strength in COPD patients practicing Liuzijue still cannot be determined.

Wu et al. [49] measured the handgrip strength of COPD patients and found that the improvement of handgrip strength in the Liuzijue group improved after 3 months. However, clinical significance (15\%) was not achieved in this study. Liu et al. [32] assessed isokinetic muscle strength of elbow and knee joint muscle groups, and the comparison parameters included the $\mathrm{PT}, \mathrm{PT} / \mathrm{BW}, \mathrm{TW}$, and ER of two groups. The results revealed that elbow extensor TW achieved the MCID (15\%). Handgrip strength and isokinetic muscle strength parameters were only reported by one study. It cannot yet be determined whether Liuzijue can improve limb muscle strength in COPD patients.

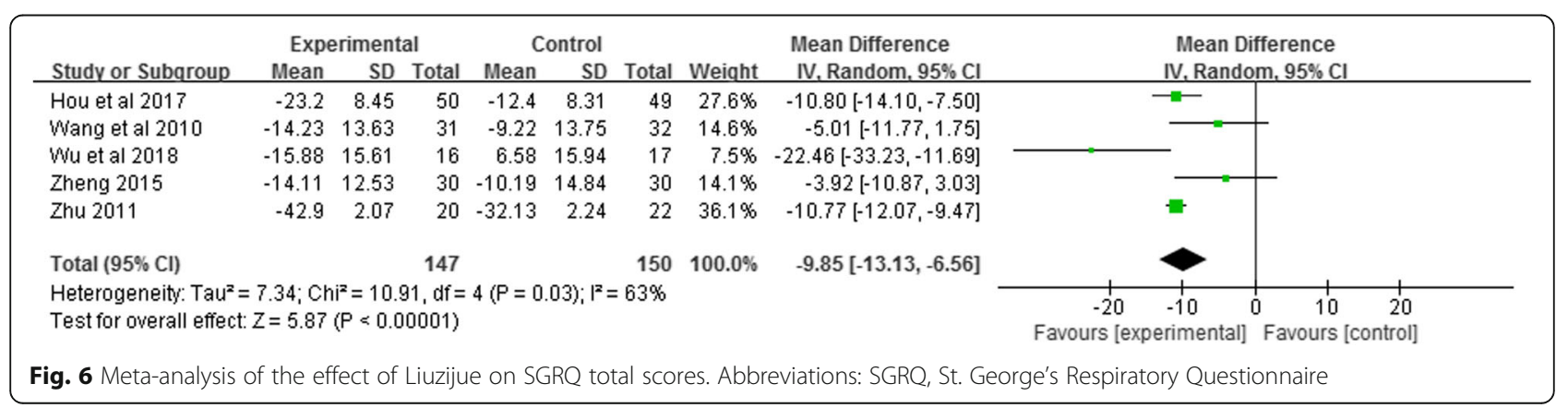




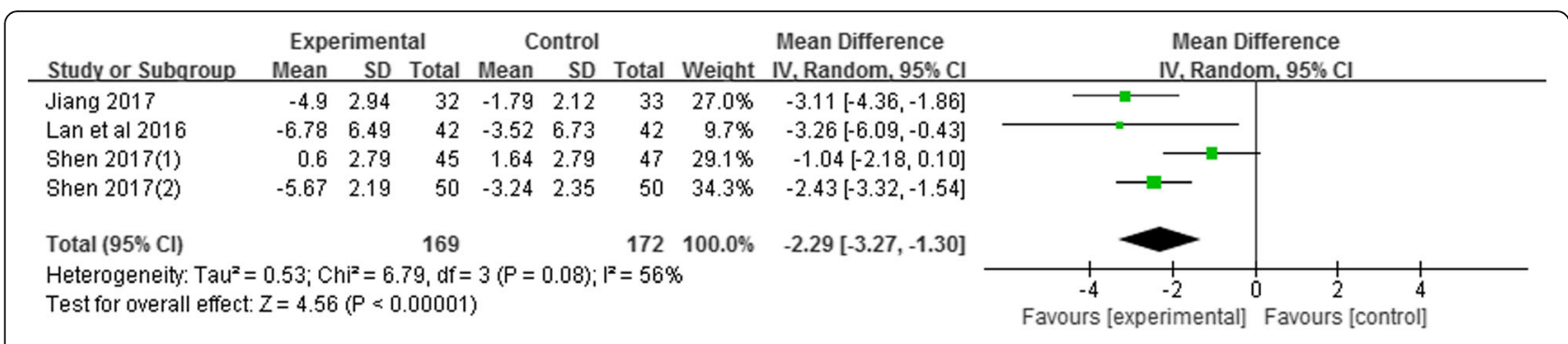

Fig. 7 Meta-analysis of the effect of Liuzijue on CAT scores. Abbreviations: CAT, Chronic Obstructive Pulmonary Disease Assessment Test

6MWD improved significantly in the Liuzijue group of $5 / 6$ studies, and 4/5 studies [39, 42, 44, 49] achieved the minimal clinical significance indicator of $25 \mathrm{~m}$ [50]. Patients practiced Liuzijue at least 5 days (200 min) a week, and training duration was 3 months (one study) or 6 months (three studies) in these four studies. Heterogeneity between the studies was not apparent, which means the results were credible. These findings indicate that Liuzijue can significantly improve the exercise endurance of COPD patients. In a study by Wu et al. [49], 30s SST was applied to evaluate the exercise ability of patients, but improvement was not notable between the two groups. Owing to the low sample size of this study and the fact that this indicator was not used in other studies, the improvement in 30s SST times is still uncertain.

\section{Lung function}

$\mathrm{FEV}_{1}$ improved significantly in the Liuzijue group of $4 / 8$ studies, and four studies [37, 40, 44, 48] achieved the MCID of $0.1 \mathrm{~L}$ [51]. Patients practiced at least $30 \mathrm{~min}$ a day, 7 days a week, and practice duration was 3 months (three studies) or 6 months (one study) in these four studies. The meta-analysis results suggest that Liuzijue exercise can significantly improve the lung function $\left(\mathrm{FEV}_{1}\right.$, $\mathrm{FEV}_{1} \%$ pred, $\mathrm{FEV}_{1} / \mathrm{FVC} \%$ ) of patients. However, because the heterogeneity between studies is obvious, the conclusions should be treated cautiously. We attempted to perform subgroup analysis for pulmonary function indicators, but the heterogeneity between the studies has not been eliminated. It may be caused by the variations in age, sex ratio, and disease severity among the studies.

\section{Quality of life}

The MCIDs of SGRQ and CAT in patients with COPD were -7.43 units and -2.54 units, respectively [56]. All studies reported SGRQ [38, 40, 42, 48, 49] and three studies reported CAT $[43,44,46]$, showing significant clinical differences between the two groups. Liuzijue practice frequency varied from 20 to $60 \mathrm{~min}$ a day, and at least 5 days (140 min) a week. Practice duration was 3 months (four studies) or 6 months (four studies) in these eight studies. Meta-analysis results showed that compared with the control group, Liuzijue exercise had a significant effect on the improvement of quality of life (CAT score, SGRQ total score, and symptom and activity scores of SGRQ) in COPD patients. However, there was significant heterogeneity among the studies. Differing disease severity in the participants and varying intervention times may be the possible reasons for this heterogeneity. Therefore, the identification of the improvement effect of Liuzijue exercise on patients' quality of life requires further research.

\section{Limitations}

Several limitations of the present study should be noted. First, as the Liuzijue exercises require active engagement, blinding of participants and staff was impossible. Additionally, a number of studies have not reported a blind method of independent outcome assessment, which could minimize the expectation bias of outcomes. This may result in an overestimation of the effect of Liuzijue on COPD patients. Second, because there was a lack of longterm follow-up studies, we cannot ensure the long-term effect of Liuzijue on COPD patients. Finally, our review only searched for relevant studies published in Chinese and English, and was unable to include all relevant research, which may lead to a results bias.

\section{Conclusion}

Our study suggested that Liuzijue could effectively improve dyspnea, exercise capacity, lung function, and quality of life for COPD patients. For those COPD patients with apparent dyspnea, Liuzijue training frequency should reach at least 150 min per week, 5 days a week. However, there is only modest information about the effect of Liuzijue on stable COPD, which primarily comes from small, inadequate studies with potential risk of bias. That bias may increase the obvious benefits of Liuzijue, which means the evidence of benefit from Liuzijue was of low quality. Also, the likelihood is high that the effect will be substantially different from the estimate in this systematic review.

In addition, we have the following suggestions for future research. First, the clinical trial methodologies of many studies, such as random sequence generation, allocation concealment, and blinded independent outcome assessment, are not rigorous enough. The safety of Liuzijue has not been reported by some studies. We hope that future 
research will be further strengthened in methodology. Second, the intervention duration in current studies is mostly either three or 6 months. Large-sample, high-quality RCTs are needed in the future to investigate the long-term effect of Liuzijue on COPD patients. Third, most existing studies only assessed exercise endurance; there is a lack of research on the effect of Liuzijue on patients' muscle strength. More studies investigating the effect of Liuzijue on patients' respiratory muscle and limb muscle strength are needed. Finally, future rehabilitation programs must be more personalized according to the severity of COPD patients.

\section{Supplementary information}

Supplementary information accompanies this paper at https://doi.org/10. 1186/s12906-020-03104-1.

Additional file 1: Table S1 Characteristics of included studies. CG = Control Group; LG = Liuzijue Group; " - "=Not reported in the study; MRC = Medical Research Council Dyspnea Scale; $\mathrm{mMRC}=$ Modified Medical Research Council Dyspnea Scale; MIP = Maximal Inspiratory Pressure; MEP = Maximal Expiratory Pressure; $6 \mathrm{MWD}=$ six-minutes walking distance; 30s SST =30s Sit-to-Stand Test; FEV1 = Forced expiratory volume in one second; FEV1\%pred = the percentage of predicted values of FEV1; FVC $=$ Forced vital capacity; $S G R Q=$ St. George's respiratory questionnaire CAT $=$ Chronic Obstructive Pulmonary Disease Assessment Test.

Additional file 2: Table S2 Adverse Events.

Additional file 3: Fig. S1 Meta-analysis of the effect of Liuzijue on SGRQ score: (A) Symptom; (B) Activity; (C) Influence. Abbreviations: SGRQ, St. George's Respiratory Questionnaire.

\section{Abbreviations}

30s SST: 30 s Sit - to - Stand Test; 6MWD: 6 min walking distance; BW: Body weight; CAT: Chronic Obstructive Pulmonary Disease Assessment Test; CG: Control group; Cl: Confidence Interval; CNKI: China Network Knowledge Infrastructure; COPD: Chronic Obstructive Pulmonary Disease; ER: Muscle endurance ratio; $\mathrm{FEV}_{1}$ : Forced expiratory volume in one second; $\mathrm{FEV}_{1} \%$ pred: Percentage of predicted values of $\mathrm{FEV}_{1}$; FVC: Forced vital capacity; LG: Liuzijue group; MD: Mean difference; MEP: Maximal expiratory pressure; MIP: Maximal inspiratory pressure; mMRC: Modified Medical Research Council Dyspnea Scale; MRC: Medical Research Council Dyspnea Scale; PT: Peak torque; RCTs: Randomized controlled trials; SGRQ: St. George's Respiratory Questionnaire; TW: Total work

\section{Acknowledgments}

The authors really appreciate the help of people from Shanghai University of Traditional Chinese Medicine and Shanghai University of Sport.

\section{Authors' contributions}

$X D L, W B W$ and $L X$ contributed to the design of this review. PJL and CLS conducted the literature search and selection. LX, HXD and PJL did the quality assessment. LX, HXD and XDL complete the data extraction. LX and HXD completed data analysis and the manuscript. All authors read and approve the final manuscript.

\section{Funding}

This review was supported by the National Natural Science Foundation of China (81902307) and the research project of Health Qigong Administrative Center of the General Administration of Sport of China (QG2019011), which supported the study design and data extraction.

\section{Availability of data and materials}

The datasets used and analyzed during the current study are available from the corresponding author upon reasonable request.
Ethics approval and consent to participate

Not applicable.

\section{Consent for publication}

Not applicable.

\section{Competing interests}

The authors declare that they have no competing interests.

\section{Author details}

${ }^{1}$ School of Rehabilitation Science, Shanghai University of Traditional Chinese Medicine, Shanghai, China. ${ }^{2}$ Department of Rehabilitation, the Second Rehabilitation Hospital of Shanghai, Shanghai, China. ${ }^{3}$ Department of Sports Medicine, Shanghai University of Sport, Shanghai, China. ${ }^{4}$ Institute of Rehabilitation Medicine, Shanghai Academy of Traditional Chinese Medicine, Shanghai, China.

Received: 15 May 2019 Accepted: 5 October 2020

Published online: 14 October 2020

\section{References}

1. Global Initiative for Chronic Obstructive Lung Disease. Global strategy for the diagnosis, management, and prevention of chronic obstructive pulmonary disease, 2019. Https://goldcopd.org/wp-content/uploads/2018/11/GOLD-2019v1.7-FINAL-14Nov2018-WMS.pdf. Accessed 25 December 2018.

2. Wang $C, X u J$, Yang $L$, et al. Prevalence and risk factors of chronic obstructive pulmonary disease in China (the China pulmonary health [CPH] study): a national cross-sectional study. Lancet. 2018;391(10131):1706-17.

3. Zhong N, Wang C, Yao W, Chen P, Kang J, Huang S, et al. Prevalence of chronic obstructive pulmonary disease in China: a large, population-based survey. Am J Respir Crit Care Med. 2007;176(8):753-60.

4. World Health Organization. The top 10 causes of death. https://www.who. int/news-room/fact-sheets/detail/the-top-10-causes-of-death, Accessed 20 December 2018

5. Zhu $B$, Wang $Y$, Jian $M$, et al. Disease burden of COPD in China: a systematic review. Int J Chron Obstruct Pulmon Dis. 2018;13:1353-64.

6. World Health Organization. World health statistics 2008. https://www.who. int/gho/publications/world_health_statistics/EN_WHS08_Full.pdf?ua=1, Accessed 7 Apr 2019.

7. Flynn RWV, Macdonald TM, Chalmers JD, et al. The effect of changes to GOLD severity stage on long term morbidity and mortality in COPD. Respiratory research. 2018;19(1):249.

8. Drummond MB, Hansel NN, Connett JE, et al. Spirometric predictors of lung function decline and mortality in early chronic obstructive pulmonary disease. Am J Respir Crit Care Med. 2012;185(12):1301-6.

9. Singer J, Yelin EH, Katz PP, et al. Respiratory and skeletal muscle strength in COPD: impact on exercise capacity and lower extremity function. J Cardiopulm Rehabil Prev. 2011:31(2):111.

10. Sidharth K, Anand R, Shyam K. Prevalence of quadriceps muscle weakness in patients with COPD and its association with disease severity. Int J Chronic Obstruct Pulmon Dis. 2015;10(1):1727-35.

11. Zeng $G$, Chen $L$, Fan $H$, et al. The relationship between steps of $6 M W T$ and COPD severity: a cross-sectional study. Int J Chronic Obstruct Pulmon Dis. 2018;14:141-8.

12. Manescu $\mathrm{V}$. The relevance of the 6 minutes walking test and of dyspnea measured with $\mathrm{mMRC}$ scale in evaluating COPD severity. Pneumologia. 2012;61(3):153-9.

13. Jones PW, Brusselle G, Negro RWD, et al. Properties of the COPD assessment test in a cross-sectional European study. Eur Respir J. 2011:38(1):29.

14. Morishitakatsu M, Nishimura K, Taniguchi $H$, et al. The COPD assessment test and St George's respiratory questionnaire: are they equivalent in subjects with COPD? Int J Chronic Obstruct Pulmon Dis. 2016;11(1):1543-51.

15. Ringbaek T, Martinez $G$, Lange $P$. A comparison of the assessment of quality of life with CAT, CCQ, and SGRQ in COPD patients participating in pulmonary rehabilitation. COPD J Chron Obstruct Pulmon Dis. 2012;9(1):4.

16. Liu X, et al. Study on relationship between the St George's respiratory questionnaire and objective indexes in patients with chronic obstructive pulmonary disease in stable period. Chin J Clin Med. 2014;3:354-6.

17. Nonato NL, Díaz O, Nascimento OA, et al. Behavior of quality of life (SGRQ) in COPD patients according to BODE scores. Arch Bronconeumol (English Edition). 2015;51(7):315-21 
18. Sarioglu N, Hismiogullari AA, Bilen C, et al. Is the COPD assessment test (CAT) effective in demonstrating the systemic inflammation and other components in COPD? Rev Port Pneumol. 2016;22(1):11-7.

19. Sciriha A, Lungaro-Mifsud S, Scerri J, et al. Health status of COPD patients undergoing pulmonary rehabilitation: a comparative responsiveness of the CAT and SGRQ. Chronic Respir Dis. 2017;14(4):352-9.

20. Martin AL, Marvel J, Fahrbach $\mathrm{K}$, et al. The association of lung function and St. George's respiratory questionnaire with exacerbations in COPD: A systematic literature review and regression analysis. Respir Res. 2016;17:40.

21. Nagai K, Makita $H$, Suzuki $M$, et al. Differential changes in quality of life components over 5 years in chronic obstructive pulmonary disease patients. Int J Chronic Obstruct Pulmon Dis. 2015;2015(1):745-57.

22. Wacker ME, Jörres RA, Annika K, et al. Assessing health-related quality of life in COPD: comparing generic and disease-specific instruments with focus on comorbidities. BMC Pulmon Med. 2016;16(1):70.

23. Liu X, Li P, Li J, et al. Home-based prescribed pulmonary exercise in patients with stable chronic obstructive pulmonary disease. J Vis Exp. 2019;(150):10 3791/59765.

24. Zhang HL, Li JS, Yu XQ, et al. An evaluation of activity tolerance, patientreported outcomes and satisfaction with the effectiveness of pulmonary daoyin on patients with chronic obstructive pulmonary disease. Int J Chron Obstruct Pulmon Dis. 2017;12:2333-42.

25. Zhang $M, X v G$, Luo $C$, Meng D, Ji Y. Qigong Yi Jinjing promotes pulmonary function, physical activity, quality of life and emotion regulation self-efficacy in patients with chronic obstructive pulmonary disease: a pilot study. J Altern Complement Med. 2016;22(10):810-7.

26. Chai J, Shi C, Chen Y, et al. Acoustographic study on the respiratory methods in six-word qigong. Shanghai J Tradit Chin Med. 1999;33(9):42-6.

27. Fitness Qigong Management Center of General Administration of Sport of China. Health qigong Yijinjing Wuqinxi Liuzijue Baduanjin: People's Sports Publishing House of China. 2005.

28. Fitness Qigong Management Center of General Administration of Sport of China. Health qigong Liuzijue: People's Sports Publishing House of China. 2003.

29. Mckeough Z, Velloso M, Lima VP, et al. Upper limb exercise training for COPD. John Wiley \& Sons, Ltd: The Cochrane Library; 2014.

30. Tarigan AP, et al. The impact of upper limb training with breathing maneuver in lung function, functional capacity, dyspnea scale, and quality of life in patient with stable chronic obstructive of lung disease. Open Access Maced J Med Sci. 2019;7(4):567-72.

31. Zhou G, Yao X, Li H, et al. Discussion on internal relations between Liuzijue and Core stability training of lumbar spine. Rehabil Med. 2016;26(4):47.

32. Wu W, Liu X, Liu J, et al. Effectiveness of water-based Liuzijue exercise on respiratory muscle strength and peripheral skeletal muscle function in patients with COPD. Int J Chronic Obstruct Pulmon Dis. 2018;13:1713-26.

33. Xiaotian $L$, Jifeng $Z$, Rachel $C$, et al. The Effects of Traditional Chinese Exercise in Patients with Chronic Obstructive Pulmonary Disease: A MetaAnalysis. PLoS One. 2016;11(9):e0161564

34. Ng BHP, Tsang HWH, Ng BFL, et al. Traditional Chinese Exercises for Pulmonary Rehabilitation: evidence from a systematic review. J Cardiopulm Rehabil Prev. 2014;34(6):367-77.

35. Liu SJ, Ren Z, Wang L, Wei GX, Zou L. Mind(-)Body (Baduanjin) Exercise Prescription for Chronic Obstructive Pulmonary Disease: A Systematic Review with Meta-Analysis [J]. Int J Environ Res Public Health. 2018;15(9): 1830.

36. Wang K, Liu S, Kong Z, Zhang Y, Liu J. Mind-Body Exercise (Wuqinxi) for Patients with Chronic Obstructive Pulmonary Disease: A Systematic Review and Meta-Analysis of Randomized Controlled Trials [J]. Int J Environ Res Public Health. 2018;16(1):72.

37. Zhang W, Chen J, Zheng G. Influence of "six-character" breathing method on exercise endurance of patients with chronic obstructive pulmonary disease in stable stage. Chin Nurs Res. 2009;23(32):2957-8.

38. Wang Z, Yang P, Tang J, et al. Effects of "six letters formula" on quality of life and syndrome of traditional Chinese medicine in patients with chronic obstructive pulmonary disease. Shanghai J Tradit Chin Med. 2010;5:54-6.

39. Zhao D, Zhou Y, Li F, et al. Rehabilitation of modified exercise prescription for patients with stable chronic obstructive pulmonary disease. Int J Respir. 2012;9:678-82.

40. Zhu Z. Health Qigong's effect on the 61 stabilized patients with chronic obstructive pulmonary disease. J Nanjing Univ Tradit Chin Med. 2011;27(3): 235-7.
41. Wang Z, Yang P, Tang J. The randomized controlled trial of TCM pulmonary rehabilitation exercise on COPD patients pulmonary function and acute exacerbation rate. J Emerg Tradit Chin Med. 2013;22(1):23-4.

42. Zheng $Y$. The research of rehabilitation effect on patients with COPD for lung and kidney deficiency type by treating winter disease in summer Acupoint sticking therapy combined with the "six Strategics" skills training. Wuhan Univ Chin Med. 2015. https://kns.cnki.net/kcms/detail/detail. aspx? dbcode $=C M F D \& d b n a m e=C M F D 201601 \&$ filename $=1015649370 . \mathrm{nh} \& \mathrm{v}=$ V2MB43GXIRZWmURWT9pAqAD8gi\%25mmd2BQTvMaw3nRzjbrO7\%25 mmd2FKLa7CJQeJVBMVUhUk5VRv. Accessed 31 Aug 2018.

43. Yang $L, X u H$, Wang $Y$, et al. Impacts of the combined therapy of Tiotropium bromide and medical exercise of the six-character formula on the living quality and pulmonary function in the patients of chronic obstructive pulmonary disease at the stable stage. World J Integr Tradit Western Med. 2016;11(10):1369-71.

44. Jiang M. Effect of strengthen Liuzijue on respiratory function of stable COPD patients with lung and spleen deficiency syndrome. Nanjing Univ Chin Med. 2017. https://kns.cnki.net/kcms/detail/detail.aspx?dbcode= CMFD\&dbname $=$ CMFD201801\&filename $=1017061746$. nh\&v $=$ guLw56 AXtqWOpEF49uigOKedrTjprFFgle1CMt\%25mmd2FBMrnyzrL3Gv1 nftGFymMPEUIn. Accessed 31 Aug 2018.

45. Qian S. The study on treating COPD stabilization (levell) in elderly patients by six strategics health exercises. J External Ther Tradit Chin Med. 2017;26(6): 3-6.

46. Qian S. A community intervention study on treating COPD stabilization (levell) in elderly patients by six strategics health exercises. Clin J Chin Med. 2017;9:25.

47. Gu R. TCM pulmonary rehabilitation exercise on randomized controlled study of pulmonary function in patients with COPD and acute attack. Yiyao Qianyan. 2017;7(22):49-51.

48. Huo M, Cui X. The observation on the simplify six-character formula on the survival quality in qi deficiency of lung and kidney syndrome patients with COPD. Guangming J Chin Med. 2017;32(24):3518-21.

49. Wu W, Liu X, Li P, et al. Effect of Liuzijue exercise combined with elastic band resistance exercise on patients with COPD: a randomized controlled trial. Evid Based Complement Altern Med eCAM. 2018;2018(6):1-12.

50. Holland $A E$, Hill CJ, Rasekaba T, et al. Updating the minimal important difference for six-minute walk distance in patients with chronic obstructive pulmonary disease. Arch Phys Med Rehabil. 2010;91(2):0-225.

51. Donohue JF. Minimal Clinically Important differences in COPD lung function. COPD: J Chron Obstruct Pulmon Dis. 2005;2(1):111-24.

52. De Torres JP, Pinto-Plata $V$, Ingenito $E$, et al. Power of outcome measurements to detect clinically significant changes in pulmonary rehabilitation of patients with COPD [J]. Chest. 2002;121(4):1092-8.

53. Cabral LF, D'Elia Tda C, Marins Dde S, et al. Pursed lip breathing improves exercise tolerance in COPD: a randomized crossover study. Eur J Phys Rehabil Med. 2015;51(1):79-88.

54. Mayer AF, Karloh M, Dos Santos K, et al. Effects of acute use of pursed-lips breathing during exercise in patients with COPD: a systematic review and meta-analysis. Physiotherapy.2018;104(1):9-17.

55. Dechman G, Wilson CR. Evidence underlying breathing retraining in people with stable chronic obstructive pulmonary disease. Phys Ther. 2004;84(12): 1189-97.

56. Alma H, de Jong C, Tsiligianni I, et al. Clinically relevant differences in COPD health status: systematic review and triangulation. Eur Respir J. 2018;52(3): 1800412 .

\section{Publisher's Note}

Springer Nature remains neutral with regard to jurisdictional claims in published maps and institutional affiliations. 